Preprint of the paper:

"Stability analysis of BEM approximate solutions in grounding analysis"

J. Gómez-Calviño, I. Colominas, F. Navarrina, M. Casteleiro (2001)

En "Computational Fluid and Soild Mechanics", 1579---1581; K.J.Bathe (Ed.); Elsevier, Oxford, UK. (ISBN: 0-08-043944-6)

http://caminos.udc.es/gmni 


\title{
Stability analysis of BEM approximate solutions in grounding analysis
}

\author{
Javier Gómez-Calviño, Ignasi Colominas *, Fermín Navarrina, Manuel Casteleiro \\ Department of Applied Mathematics. Civil Engineering School, Universidade da Coruña, Campus de Elviña, 15192 La Coruña, Spain
}

\begin{abstract}
In this paper we present a summary of the Boundary Element Method developed for the analysis of potential problems in the electrical engineering field. The numerical model proposed is shown as a general frame so that other existing computer methods are recognized as particular cases. The stability of these models is analyzed identifying the sources of error.
\end{abstract}

Keywords: Grounding analysis; BEM; APM; Stability

\section{Introduction}

As it is known, a grounding system comprises all the interconnected grounding facilities of an specific area, being the grounding grid its main element. In general, most of grounding grids of electrical substations consist of a mesh of interconnected cylindrical conductors, horizontally buried, and supplemented by vertically thrusted ground rods in certain places of the substation site. The most important parameters that are required to design a safe grounding system are the potential distribution on earth surface when a fault current is derived into the soil and the equivalent resistance of the system [1,2].

Equations governing the electrical current dissipation into the soil through a grounded electrode are well-known and can be stated from Maxwell's Electromagnetic Theory. Nevertheless, their application and resolution in practical cases for grounding grids of large installations present some troubles [1] (and, of course, no analytical solutions can be obtained in a real problem).

Since the 1960s, several methods for the grounding analysis and design of electrical substations have been proposed. These methods (generally based on professional practice, semi-empirical works, experimental data obtained from scale model assays, or intuitive ideas) represented an important improvement in the grounding analysis field.

\footnotetext{
*Corresponding author. E-mail: colominas@iccp.udc.es; Web page: http://www.udc.es/caminos/dmmr
}

However, some problems (such as large computational requirements, unrealistic results when segmentation of conductors is increased, and uncertainty in the margin of error) have been reported [2].

On the other hand, widespread numerical techniques commonly used in other fields of engineering (such as the FEM and the FDM) are precluded in grounding analysis, due to the huge mesh resultant of the discretization of the soil, around the cylindrical conductors and covering semi-infinite domains [3].

\section{Mathematical model}

The problem can be stated by means of Maxwell's equations. Restricting the analysis to the electrokinetic steady-state response and neglecting the inner resistivity of the earthing conductors (potential is assumed constant on the electrode surface), the $3 \mathrm{D}$ problem can be written as

$\operatorname{div}(\boldsymbol{\sigma})=0 ; \quad \boldsymbol{\sigma}=-\boldsymbol{\gamma} \operatorname{grad}(V)$ in $E ; \quad \boldsymbol{\sigma}^{t} \boldsymbol{n}_{E}=0$ in $\Gamma_{E} ;$

$V=V_{\Gamma}$ in $\Gamma ; \quad V \rightarrow 0, \quad$ if $|x| \rightarrow \infty$,

with $E$ the earth, $\gamma$ its conductivity tensor, $\Gamma_{E}$ the earth surface, $\boldsymbol{n}_{E}$ its normal exterior unit field and $\Gamma$ the electrode surface. Therefore, when the electrode attains a voltage $V_{\Gamma}$ (Ground Potential Rise, or GPR) relative to a distant grounding point, the solution to problem Eq. (1) makes possible to obtain the potential distribution on the earth 
surface, the total surge current and the equivalent resistance of the grounding system $[1,3,4]$. Since $V$ and $\sigma$ are proportional to the GPR value, the normalized boundary condition $V_{\Gamma}=1$ is not restrictive at all, and it will be used from here on.

If the soil is considered homogeneous and isotropic, the conductivity tensor $\boldsymbol{\gamma}$ can be substituted by an experimentally obtained scalar value $\gamma$. Nevertheless, if the electrical properties of the soil change in the surroundings of the electrical installation, it seems advisable to develop more advanced soil models: a frequently proposed practical soil model consists of assuming the soil stratified in a number of horizontal layers, defined by their thicknesses and their apparent scalar conductivities. In fact, it is widely accepted that two-layer soil models should be sufficient to obtain safe designs of grounding systems in most practical cases [1]. Since the kind of techniques described in this paper can be extended to multi-layer soil models, further discussion is restricted to uniform soils.

As the surroundings of a substation site are levelled and regularized during its constructive phase, the earth surface $\Gamma_{E}$ can be assumed horizontal. Now, the application of the "method of images" and Green's Identity to problem Eq. (1) yields the following integral expression [3,4] for potential $V(\boldsymbol{x})$ at $\boldsymbol{x} \in E$ :

$V(\boldsymbol{x})=\frac{1}{4 \pi \gamma} \iint_{\xi \in \Gamma} k(\boldsymbol{x}, \boldsymbol{\xi}) \sigma(\boldsymbol{\xi}) \mathrm{d} \Gamma, \quad \forall \boldsymbol{x} \in E$,

where $\sigma(\boldsymbol{\xi})$ is the leakage current density $\left(\sigma=\boldsymbol{\sigma}^{t} \boldsymbol{n}\right.$, being $\boldsymbol{n}$ the normal exterior unit field to $\Gamma$ ) at any point $\boldsymbol{\xi}$ of the electrode surface $\Gamma \subset E$, and with the weakly singular kernel

$k(\boldsymbol{x}, \boldsymbol{\xi})=\left(\frac{1}{r(\boldsymbol{x}, \boldsymbol{\xi})}+\frac{1}{r\left(\boldsymbol{x}, \boldsymbol{\xi}^{\prime}\right)}\right), \quad r(\boldsymbol{x}, \boldsymbol{\xi})=|\boldsymbol{x}-\boldsymbol{\xi}|$,

where $\xi^{\prime}$ is the symmetric of $\boldsymbol{\xi}$ with respect to the earth surface. Since Eq. (2) holds on the earthing electrode surface, the boundary condition $V_{\Gamma}=1$ leads to a Fredholm integral equation of the first kind on $\Gamma$, the solution of which is the unknown leakage current density $\sigma$. That equation can be written in the weaker variational (or weighted residuals) form

$$
\iint_{\boldsymbol{\chi} \in \Gamma} w(\boldsymbol{\chi})\left(1-\frac{1}{4 \pi \gamma} \iint_{\boldsymbol{\xi} \in \Gamma} k(\boldsymbol{\chi}, \boldsymbol{\xi}) \sigma(\boldsymbol{\xi}) \mathrm{d} \Gamma\right) \mathrm{d} \Gamma=0,
$$

which must hold for all members $w(\chi)$ of a suitable class of so-called test (or weighting) functions on $\Gamma[3]$.

\section{BEM numerical model}

Let us take a given set of $\mathcal{N}$ so-called trial (or interpolating) functions defined on $\Gamma$, a given set of $\mathcal{M} 2 \mathrm{D}$ boundary elements (portions of the electrode surface), and a given set of $\mathcal{N}$ test functions defined on $\Gamma$ [3]. The unknown leakage current density $\sigma$ and the electrode surface $\Gamma$ can be discretized in that way. With this, it is possible to derive the corresponding discretized form for the potential Eq. (2) and for the integral equation Eq. (4). And so the approximate solution to the problem can be obtained from solving a system of linear equations $[3,4]$, as usual in BEM models. The coefficients of the matrix of that system (which is full) are the result of double 2D integrations; besides, the number of degrees of freedom is large. It can be easily understood that it is necessary to make additional assumptions in order to overcome the problem complexity.

The final form of the approximated 1D Boundary Element Formulation is obtained after accepting three hypotheses [3]: (A) the leakage current is assumed uniform around the perimeter of every cross section of the cylindrical conductor, (B) the ends and junctions of the conductors are not taken into account, and (C) the distances implied in the kernel of the integrals can be approximated by an expression that only depends on the distance between the projections of those points over the axis of the elements (in fact, that expression is the product of a quadrature that saves us integrating along the circumference, combining this with hypothesis A).

After applying these hypotheses, the coefficients of the matrix of the system to solve are the result of double 1D integrations; besides, the number of degrees of freedom (points on the axis of the elements) is lower than in the previous 2D model.

One fundamental difference between the $2 \mathrm{D}$ and the $1 \mathrm{D}$ discretization models is that in the latter one the integral kernels are non-singular [3].

\section{Stability analysis}

This stability analysis of the model implies studying its behavior when the number of degrees of freedom $n$ is increased (by refining the segmentation of the conductors): we expect that the discretized leakage current density and the discretized potential will converge to their exact solutions $\sigma(\boldsymbol{\xi})$ and $V(\boldsymbol{x})$ as $n$ rises. We can increase $n$ either by increasing the segmentation of the conductors, or by using higher order elements.

The result of this analysis is that these formulations fail to converge to the exact solution, since the discretized leakage current density becomes polluted by increasing numerical instabilities when discretization is refined beyond a certain point, although the equivalent resistance seems to converge [3]. We will try to find out the causes of those instabilities, by analyzing the three hypotheses (A, B, C, see above) $[3,4]$ introduced to overcome the computational complexity of the 2D BEM general formulation. Assumptions $\mathrm{A}$ and $\mathrm{B}$ are discarded as causes of the instabilities: several numerical tests made by the authors [3] with a 
single bar prove that hypothesis A is safe to assume; on the other hand, experience has shown that assumption B could affect the results around the ends or the joints of the electrodes, but it does not seem to compromise the global behavior of the model.

So the origin of the instabilities must be attributed to the assumption $\mathrm{C}$. The fact is that the mentioned approximations are not valid for short distances. When the discretization is refined, the size of the segments become comparable to or smaller than the diameter of the conductors. Then, approximation $\mathrm{C}$ introduces significant errors in the coefficients of the linear system, particularly in the diagonal terms: these terms correspond to coupling points of the same elements, and so can be very affected by the bad approximation of the circumferential integral, as the distances are all very short. On the other hand, when the ratio diameter/length of the elements is small, the bad approximations due to short distances are hidden by the dominant good approximations, as the elements are long enough. From another point of view: it is a known theoretical result for Fredholm equations of the first kind that the inverse of a completely continuous operator is unbounded [5]; in plain words: if approximation $\mathrm{C}$ is used, the exact solution of the ill-conditioned simplified problem can not be found numerically, since one can always come upon very different leakage current distributions that apparently verify the boundary condition $V=V_{\Gamma}$ with arbitrarily small errors. This explains why unrealistic results are obtained when discretization is refined, and convergence is precluded.

\section{Application to real cases}

The techniques derived by the authors have been implemented in a computer aided design system for earthing grids of electrical substations. At present, the single-layer code runs in real-time in personal computers. Some of these results can be found in [3,4]. The techniques described in this paper can be extended for multi-layer soil models, although computing time becomes not contemptible whatsoever. The proposed formulation has been implemented in a high-performance parallel computer and the code has been applied to the analysis of several real grounding systems [6].

\section{Acknowledgements}

This project is partially supported by the Ministerio de Ciencia y Tecnología (\#1FD97-0108) of the Spanish Government, cofinanced with FEDER funds, by the power company Uníon Fenosa Ingeniería S.A., and by research fellowships of the Xunta de Galicia and the Universidad de La Coruña.

\section{References}

[1] ANSI/IEEE Std.80. Guide for Safety in AC Substation Grounding; Draft Guide for Safety in AC Substation Grounding. New York: IEEE, 1999.

[2] Sverak JG, Dick WK, Dodds TH, Heppe RH. Safe substation grounding. IEEE Trans Power Appar Systems 1981;100:4281-4290 (Part 1); 1982;101:4006-4023 (Part II).

[3] Colominas I. A CAD System for Grounding Grids in Electrical Installations: A Numerical Approach Based on the Boundary Element Integral Method. Ph.D. Thesis, Universidade da Coruña, 1995.

[4] Colominas I, Navarrina F, Casteleiro M. A boundary element numerical approach for grounding grid computation. Comput Methods Appl Mech Eng 1999;174:73-90.

[5] Kolmogorov AN, Fomin SV. Introductory Real Analysis. New York: Dover Publications, 1975.

[6] Gómez-Calviño J, Colominas I, Navarrina F, Casteleiro M, Cela JM. Parallel computing aided design of earthing systems for electrical substations in non homogeneous soil models. In: Sadayappan P (Ed), Proceedings of the 2000 ICPP Workshops. IEEE Comp Society Press, pp. 381-388, 2000. 